\title{
Monitoring the hydration of MgO-based cement and its mixtures with Portland cement by 'H NMR relaxometry
}

Francesca Martini, ${ }^{\text {abb }}$ Silvia Borsacchi, ${ }^{b,}$ Marco Geppi, ${ }^{a, b}$ Monica Tonelli, Francesca Ridi, Lucia Calucci ${ }^{\text {bo }}$

Dipartimento di Chimica e Chimica Industriale, Università di Pisa, via G. Moruzzi 13, 56124 Pisa Italy

Istituto di Chimica dei Composti OrganoMetallici, Consiglio Nazionale delle Ricerche - CNR, S.S. di Pisa, via G. Moruzzi 1, 56124 Pisa, Italy

'Dipartimento di Chimica "Ugo Schiff” \& CSGI, Università di Firenze, via della Lastruccia 3, 50019 Sesto Fiorentino (FI), Italy

Corresponding authors: Silvia Borsacchi, Istituto di Chimica dei Composti OrganoMetallici, Consiglio Nazionale delle Ricerche - CNR, S.S. di Pisa, via G. Moruzzi 1, 56124 Pisa, Italy. Phone: +39-0503153052, e-mail: silvia.borsacchi@pi.iccom.cnr.it; Lucia Calucci, Istituto di Chimica dei Composti OrganoMetallici, Consiglio Nazionale delle Ricerche - CNR, S.S. di Pisa, via G. Moruzzi 1, 56124 Pisa, Italy. Phone: +39-0503152517, e-mail: lucia.calucci@pi.iccom.cnr.it 


\section{Abstract}

The hydration process of MgO-based cement, Portland cement, and several mixtures of them was investigated for 1 month by measuring ' $\mathrm{H}$ transverse relaxation times $\left(\mathrm{T}_{2}\right)$ of the different fractions of water inside pastes by means of the Carr-Purcell-MeiboomGill pulse sequence. The obtained $\mathrm{T}_{2}$ distributions were exploited to get information on the kinetics of the hydration process and on the evolution of the porous structure of the calcium silicate hydrate (C-S-H) and magnesium silicate hydrate (M-S-H) binder phases as a function of time. The analysis of solid echo $\mathrm{H}$ Free Induction Decays was employed to determine the relative amount of hydrogen-containing solid phases present in pastes at the longest hydration time.

Keywords: Proton relaxation times, calcium silicate hydrate, magnesium silicate hydrate, fumed silica, cement hydration. 


\section{Introduction}

In recent years, $\mathrm{MgO}$-based cements have been proposed both as potential low- $\mathrm{CO}_{2}$ alternatives to Portland cement, the dominant form of cement used worldwide, and as low-pH cements for nuclear waste immobilization [1].

The binder phase of these cements is magnesium silicate hydrate (M-S-H), the amorphous phase that forms from the reaction of $\mathrm{MgO}$ with a source of silica and water. Although a significant quantity of literature exists concerning the structure and nature of the $\mathrm{M}-\mathrm{S}-\mathrm{H}$ gel $[2,3,4,5,6,7]$, the understanding of $\mathrm{M}-\mathrm{S}-\mathrm{H}$ as a binder phase is in its infancy. In particular, questions are still open concerning the hydration of $\mathrm{MgO} / \mathrm{SiO}_{2}$ mixtures, even if this process is fundamental for the formation of the binder phase, ultimately determining microstructural properties, such as composition, porosity, and water distribution, which in turn control the mechanical properties of cement. In recent papers, the inferior mechanical properties of $\mathrm{MgO}$-based cements with respect to traditional $\mathrm{CaO}$-based cements have been correlated to the different structures of the respective binder phases (i.e M-S-H and C-S-H (calcium silicate hydrate)) at the nanoand micro-metric scale [8], as well as to the different distribution of water inside them [9]. In particular, it has been shown that poly-disperse spherical globules (without an internal layered structure) packed into a fractal structure constitute $\mathrm{M}-\mathrm{S}-\mathrm{H}$ at variance with C-S-H, where, according to the Jennings model [10], colloidal disc-like globules with a layered substructure including water pack in flocs. Moreover, the higher compressive strength of $\mathrm{C}-\mathrm{S}-\mathrm{H}$ with respect to $\mathrm{M}-\mathrm{S}-\mathrm{H}$ has been associated to a higher amount of structural and constrained water, the latter being in turn considered as an indication of a smaller average pore size.

The hydration of cement is a very complex process in which reactants dissolve in water and hydrated phases precipitate to form gels with a porous structure that evolves in time. In the case of Portland cement, calcium silicates (alite and belite) and aluminates dissolve and C-S-H forms together with Portlandite and minor amounts of calciumaluminate phases. In the case of $\mathrm{MgO} / \mathrm{SiO}_{2}$ mixtures, $\mathrm{MgO}$ dissolves giving rise to $\mathrm{Mg}^{2+}$ 
and $\mathrm{OH}$ ions; when a saturation concentration is reached, brucite precipitates and a moderately basic $\mathrm{pH}$ is established, which favors the hydration of silica with formation of silicate anions. From the reaction between silicate anions and $\mathrm{Mg}^{2+}$ cations $\mathrm{M}-\mathrm{S}-\mathrm{H}$ forms at the expense of brucite and silica [3].

'H NMR relaxometry, through the non invasive measurement of transverse $\left(\mathrm{T}_{2}\right)$ and/or longitudinal $\left(\mathrm{T}_{1}\right)$ relaxation time(s) of protons directly on as prepared pastes, gives unique information on the kinetics of formation of hydrogen-containing solid phases and on the evolution of the state of water and the pore network during cement hydration $[11,12,13,14]$. In particular, measurements and analyses of 'H Free Induction Decay (FID) on Portland cement pastes allowed protons in solid phases to be singled out and quantified $[15,16,17]$. Moreover, the dependence of the $T_{2}$ values of protons of water inside pores on the surface-to-volume ratio of pores and on a specific surface relaxivity has been exploited to distinguish and quantify different water populations $[18,19,20$, 21]. Under the assumption of fast diffusion conditions and fully saturated pores [22, 23], the distribution of pore sizes in the C-S-H gel and its evolution in the different stages of cement hydration have been determined [24, 25].

In the present work, 'H NMR relaxometry is employed for the first time to investigate the hydration process of $\mathrm{MgO}$-based cements. To this aim, $\mathrm{T}_{2}$ measurements were performed using the Carr-Purcell-Meiboom-Gill (CPMG) pulse sequence [26] at different times, ranging from $\sim 0.5 \mathrm{~h}$ to $\sim 1$ month, during the hydration of a 1:1 molar mixture of highly reactive periclase and fumed silica, either alone (MS100) or mixed with different proportions of ordinary Portland cement (MS80C20, MS50C50, and MS20C80). The hydration of the sole Portland cement (C100), carried out under the same conditions, was also investigated for comparative purposes. Moreover, 'H FID analyses on pastes of all samples hydrated for $\sim 1$ month were exploited to highlight the presence of hydrogen-containing solid phases. The results were discussed taking into account findings on the nature and structure of the hydrated phases obtained by other techniques $[27,28]$. 


\section{Experimental}

'H NMR measurements were performed at $20.7 \mathrm{MHz}$ and $24{ }^{\circ} \mathrm{C}$ using a Niumag permanent magnet interfaced with a Stelar PCNMR console. The temperature was controlled within $\pm 0.1^{\circ} \mathrm{C}$ with a Stelar VTC90 variable temperature controller. The $90^{\circ}$ pulse duration was $3 \mu \mathrm{s}$. CPMG measurements [26] were carried out with an echo time $(\tau)$ of $20 \mu$ s and acquiring 120 scans. Solid echo experiments were performed with an echo delay of $14 \mu$ s and acquiring 120 scans. The recycle delay was $2 \mathrm{~s}$.

For the NMR measurements, pastes prepared as described in the Supplementary material were deposited at the bottom of $5 \mathrm{~mm}$ NMR tubes and tightly sealed with Parafilm. Samples were stored at $24 \pm 1{ }^{\circ} \mathrm{C}$ in a temperature-controlled room during hydration. Measurements were performed at different hydration times and the reproducibility of the results was tested by comparing measurements on at least two independently prepared samples.

Relaxation decay data from CPMG experiments were inverted to give $\mathrm{T}_{2}$ distributions by the algorithm UPEN [29,30] implemented in UpenWin 1.05 software [31]. Experimental FIDs acquired on resonance using the solid echo pulse sequence were fitted to a linear combination of a Gaussian and one or two exponential functions using a non-linear least squares procedure implemented in the Mathematica [32] environment. Solid echo FIDs were used to determine the fraction of protons arising from reacted water in solid phases (given by the fractional weight of the Gaussian function) and the corresponding $\mathrm{T}_{2}$ value. The longer exponential decay(s), ascribable to unreacted water with different degree of mobility, and more affected by magnetic field inhomogeneity, were instead determined by the CPMG experiments.

\section{Results}

Representative examples of $\mathrm{T}_{2}$ distributions obtained from CPMG data are shown in Figure 1 for all samples; vertical expansions are reported in Figure S1. When well- 
separated $T_{2}$ peaks are observed, trends of $T_{2}$ values at the maxima and corresponding proton populations are also reported as a function of the hydration time, $t_{\mathrm{H}}$, in Figure 2 and $\mathrm{S} 2$, respectively.

For $\mathrm{C} 100$, at $\mathrm{t}_{\mathrm{H}}<6 \mathrm{~h}$ two $\mathrm{T}_{2}$ peaks centered at $\sim 3$ and $12 \mathrm{~ms}$ with relative weights of $\sim$ 20 and $80 \%$ are observed (Figure 1), both ascribable to "free" water within the interstices between clinker grains $[15,24]$; in the following, the fractions of water associated to these peaks will be referred to as "less mobile" and "more mobile" free water, respectively. By increasing $t_{\mathrm{H}}$, the more mobile water peak progressively broadens and shifts to shorter $\mathrm{T}_{2}$; at $\mathrm{t}_{\mathrm{H}}=8 \mathrm{~h}$ the peak is centered at $7 \mathrm{~ms}$ and partially overlaps with that of less mobile water. For longer $t_{\mathrm{H}}$ values the two peaks merge in a broad one centered at $\sim 4 \mathrm{~ms}$, whose relative intensity and $\mathrm{T}_{2}$ progressively decrease up to $\sim 30 \mathrm{~h}$ (Figures 1 and 2). Afterwards, $\mathrm{T}_{2}$ remains approximately constant at $\sim 2.5 \mathrm{~ms}$, a value comparable with those previously ascribed to water in the spaces between $\mathrm{C}-\mathrm{S}$ $\mathrm{H}$ agglomerates (interhydrate water $[25,36]$ ). After one day, an additional very broad $\mathrm{T}_{2}$ distribution shows up in the range between 0.1 and few $\mathrm{ms}$, ascribable to water in C-S$\mathrm{H}$ gel pores and interlayer spacings [24]. Further $\mathrm{t}_{\mathrm{H}}, \mathrm{a}_{2}$ peak centered at $\sim 0.1 \mathrm{~ms}$, due to water in C-S-H interlayer spaces, starts to be resolved, its relative intensity increasing with $\mathrm{t}_{\mathrm{H}}$.

For MS20C80, three peaks centered at $\sim 0.5,3$, and $8 \mathrm{~ms}$ and with relative areas of $12 \%, 23 \%$, and $65 \%$ are observed for $\mathrm{t}_{\mathrm{H}}<2 \mathrm{~h}$ (Figures 1 and 2). As for C100, the two peaks at longer $T_{2}$ can be ascribed to free water $[15,24]$. On the other hand, the peak at the shortest $T_{2}$ can be associated to water inside powder grain agglomerations ("embedded" water $[12,20,33])$ and, in particular, to water embedded within fumed silica aggregates [34]. Indeed, an analogous peak is observed at early hydration times in the $\mathrm{T}_{2}$ dispersions of all samples but $\mathrm{C} 100$. After $\sim 3 \mathrm{~h}$ the two peaks of free water converge in a broad peak, whose maximum $T_{2}$ value and intensity decrease with increasing $t_{\mathrm{H}}$. At the same time, the peak of embedded water broadens and, when still present, subsequently overlaps with the $\mathrm{T}_{2}$ distribution due to water in $\mathrm{C}-\mathrm{S}-\mathrm{H}$, which is 
the main hydrated phase formed in this sample (vide infra). After one day of hydration the evolution of the $\mathrm{T}_{2}$ distribution resembles that observed for $\mathrm{C} 100$.

For $\mathrm{t}_{\mathrm{н}}<4 \mathrm{~h}$, MS50C50 shows $\mathrm{T}_{2}$ distributions analogous to those of MS20C80, whereas the $T_{2}$ distributions of the two samples are substantially different for $t_{\mathrm{H}}$ between $4 \mathrm{~h}$ and 5 days (Figures 1, 2 and $\mathrm{S} 2$ ). In the latter period a bimodal $\mathrm{T}_{2}$ distribution is observed for MS50C50 with a peak centered at $\sim 0.5 \mathrm{~ms}$ at all times and one moving from 5 to 2 ms with increasing $t_{\mathrm{H}}$. From 5 days a broad distribution with $\mathrm{T}_{2}$ values ranging from 0.2 to $\sim 10 \mathrm{~ms}$ is observed, a peak centered at $\sim 0.4 \mathrm{~ms}$ progressively emerging with increasing $\mathrm{t}_{\mathrm{H}}$. In analogy with $\mathrm{C} 100$ and MS20C80, this distribution can be ascribed to gel pores and interlayer water in $\mathrm{C}-\mathrm{S}-\mathrm{H}$, which is the main phase formed also for this sample (vide infra).

Samples MS100 and MS80C20 show similar $\mathrm{T}_{2}$ distributions with three well-separated peaks for hydration times up to 1 day (see Figures 1, 2, and S2). In particular, at the beginning of hydration, the three peaks are centered at $\sim 0.4,3$ and $8 \mathrm{~ms}$ for both samples and account for 10 (12), 10 (13), and 80 (75)\% of water protons for MS100 (MS80C20). The peak at the shortest $\mathrm{T}_{2}$ can be ascribed to embedded water, as for the previous samples, while the other peaks should be due to free water with different mobility in the spaces between powder grains. With increasing $t_{\mathrm{H}}$, the embedded water peak broadens, but its maximum remains almost at the same $T_{2}$ value and its intensity essentially does not change (see insets of Figure 1). The peak of less mobile free water also broadens and does not show significant changes in the $T_{2}$ maximum value, but its intensity slightly increases. On the other hand, the peak of more mobile water moves to shorter $\mathrm{T}_{2}$ values and slightly decreases in intensity. For MS100, after $\sim 30 \mathrm{~h}$ the $\mathrm{T}_{2}$ maximum values of the three peaks remain practically constant at $\sim 0.4,2.8$, and 4.3 $\mathrm{ms}$; the only change observed consists in the broadening of the two peaks at lower $\mathrm{T}_{2}$. On the contrary, for MS80C20 after 1 day the two peaks of free water merge in a broad one, whose maximum $T_{2}$ progressively decreases with increasing $t_{н}$ up to 6 days and 
then it stabilizes at $\sim 1.7 \mathrm{~ms}$. The peak at $\sim 0.4 \mathrm{~ms}$ progressively broadens with increasing $\mathrm{t}_{\mathrm{H}}$.

After about 1 month, solid echo 'H FIDs of all samples show the presence of a solid component characterized by a Gaussian decay with $\mathrm{T}_{2}$ of $12,19,14,18$, and $18 \mu$ s that accounts for 24, 9, 5, 5, and 5\% of protons for C100, MS20C80, MS50C50, MS80C20, and MS100, respectively.

\section{Discussion}

Data here acquired by 'H NMR relaxometry can be interpreted to get insights into the kinetics of the hydration process and the evolution of the porous structure of the hydrated phases with time, also taking into account complementary results obtained by other techniques (i.e. X-ray diffraction (XRD), thermogravimetric analysis (DTG), differential scanning calorimetry (DSC), and ${ }^{2} \mathrm{Si}$ solid state NMR) on the same samples $[27,28]$. As reported in Ref [28], Free Water Index (FWI) trends obtained by DSC measurements [35] showed three steps of the hydration process: an induction period (from 30 minutes to few hours after mixing) with low water consumption; a "nucleation and growth" period (including the acceleration and deceleration stages), lasting until 30 - $40 \mathrm{~h}$, during which water is rapidly consumed; a diffusional period characterized by a very low rate of water consumption. From XRD, DTG and ${ }^{2} \mathrm{Si}$ NMR data acquired on freeze-dried samples [2, 27, 28], we found that mainly M-S-H forms for MS80C20, while C-S-H is essentially the only phase formed for MS20C80; for MS50C50 both phases are present even if C-S-H is largely prevalent. Obviously, only M-S-H and C-S$\mathrm{H}$ form in samples MS100 and C100, respectively. For all samples hydrated phases are already present after $24 \mathrm{~h}$, although their formation is much faster for the mixed samples with respect to C100 and MS100, and reactants are almost completely consumed after 28 days of hydration. Brucite also forms for all $\mathrm{MgO}$ containing samples, while Portlandite is found only for samples C100 and MS20C80. 
In the present work, in agreement with FWI results, the three stages of hydration above described [35] can be distinguished in the monitored period (from $\sim 0.5 \mathrm{~h}$ to $\sim 1$ month) by following the evolution of the $T_{2}$ distributions as a function of $t_{\mathrm{H}}$.

For C100 the trend of the more mobile water $\mathrm{T}_{2}$ as a function of $\mathrm{t}_{\mathrm{H}}$ (Figure 2 ) is in agreement with those previously reported in the literature for white and ordinary Portland cements $[15,20,21,25,36]$. Up to $\sim 2 \mathrm{~h} \mathrm{~T}_{2}$ is practically constant: this corresponds to the induction period characterized by the dissolution of the calcium silicates, with slow release of calcium and hydroxide ions. The second stage, between 2 and $30 \mathrm{~h}$, is characterized by a rapid decrease of the more mobile water $\mathrm{T}_{2}$ with increasing $t_{H}$. For $t_{H}>8 \mathrm{~h}$ a sole $T_{2}$ peak is observed for free water centered at a value further decreasing with increasing $t_{\mathrm{H}}$. This stage corresponds to the nucleation and growth period of cement hydration, during which $\mathrm{C}-\mathrm{S}-\mathrm{H}$ and Portlandite form; C-S-H fills the spaces between the cement grains, thus confining water in progressively smaller pores associated with shorter $T_{2}$ values. During the diffusional period $\left(t_{\mathrm{H}}>30 \mathrm{~h}\right)$, the $\mathrm{T}_{2}$ of free water stabilizes at a value of $\sim 2 \mathrm{~ms}$, typical of interhydrate water $[25,36]$. At the end of the deceleration period and during the diffusional one, the progressive formation of the $\mathrm{C}-\mathrm{S}-\mathrm{H}$ gel pores and interlayer spaces is indicated by the appearance of a broad $\mathrm{T}_{2}$ distribution between 0.1 and few ms (Figure 1).

For MS100 the progressive decrease of $\mathrm{T}_{2}$ of the more mobile free water peak observed up to $40 \mathrm{~h}$ (Figure 2), accompanied by a concomitant slight decrease of its relative proton population (Figure S2), can be associated to the nucleation and growth period, during which water is consumed and M-S-H forms filling the interstitial spaces between the powder grains. Differently from Portland cement, the induction period seems to be quite short, since the decrease of $T_{2}$ already starts within the first hour of hydration. The progressive broadening of the peaks of less mobile free water and embedded water with increasing $\mathrm{t}_{\mathrm{н}}$ can be due to changes of the paste microstructure associated with the formation of M-S-H. From previous studies on MS100 [7, 27, 28], it is known that the formation of M-S-H continues between 7 and 28 days; however, the fact that the $\mathrm{T}_{2}$ 
values and the weights of the different water fractions remain practically constant after $40 \mathrm{~h}$ indicates that the average sizes and the relative amounts of the main pore populations do not significantly evolve.

The $T_{2}$ distributions obtained for the mixtures can be discussed in comparison with those of MS100 and C100 to highlight mutual effects of the reactants on the hydration process. In the case of MS80C20, for which M-S-H is mainly formed, the evolution of the $T_{2}$ distributions with increasing $t_{\mathrm{H}}$ is analogous to that observed for MS100 (Figures 1 and 2), but with three noticeable differences: the induction period seems to be slightly longer; a single free water population is observed after about one day of hydration; the $\mathrm{T}_{2}$ of free water decreases until over $100 \mathrm{~h}$ of hydration, suggesting a corresponding decrease of pore sizes for a longer time with respect to MS100. These differences can be at least partially ascribed to the formation of small amounts of C-S-H, as detected by XRD $[27,28]$. In the case of MS20C80, for which C-S-H is the main binder phase, the evolution of the $T_{2}$ dispersions with increasing $t_{\mathrm{H}}$ is similar to that observed for C100, suggesting an analogous development of the gel structure. However, the hydration kinetics is accelerated in both the induction and nucleation and growth periods, in agreement with findings from FWI measurements and structural characterizations [27, 28]. This is most probably due to the presence of fumed silica, which can both act as nucleating agent for the formation of C-S-H and form additional C-S-H through the pozzolanic reaction with Portlandite $[37,38,39,40]$. For MS50C50 the evolution of the $\mathrm{T}_{2}$ distributions with hydration time cannot be straightforwardly associated to that of either C100 or MS100 (Figures 1, 2 and S2). Indeed, the induction period is very short, as for MS100, but the hydration kinetics in the nucleation and growth period is accelerated. Moreover, differently from all the other samples, $\mathrm{T}_{2}$ of free water remains constant and practically no evolution of the $\mathrm{T}_{2}$ distributions is observed for a quite long period (from 6 to $30 \mathrm{~h}$ ). On the other hand, after $\sim 30 \mathrm{~h}, \mathrm{~T}_{2}$ distributions resemble those observed for C100 and MS20C80, in agreement with the fact that also for this sample $\mathrm{C}-\mathrm{S}-\mathrm{H}$ is the main phase formed. 
Considering that, on the basis of XRD and ${ }^{2} \mathrm{Si}$ NMR findings, in all cases reactants are almost completely consumed at 28 days of hydration, the results of ' $\mathrm{H}$ solid echo FID analyses can be exploited to compare the amount of reacted water among the different samples. For Portland cement the fast decaying Gaussian component of the FID can be mainly ascribed to Portlandite and/or to Ettringite crystal water [15, 17, 24, 25], with minor contributions from $\mathrm{OH}$ groups and bound water in C-S-H, whereas for MS100 it can be associated to $\mathrm{OH}$ groups of brucite and/or M-S-H. This considered, the decrease of the relative amount of protons in the solid phases observed going from C100 to MS20C80 and MS50C50 can be accounted for by the decrease of Portlandite content observed by XRD experiments, Portlandite being consumed in the pozzolanic reaction with silica. For MS100 and MS80C20, the equal contributions of solid phase protons to the FIDs are in agreement with the similar amounts of brucite and M-S-H and the absence of Portlandite in MS80C20 found by XRD measurements.

For samples hydrated for 28 days, $\mathrm{T}_{2}$ distributions (Figure 1 and $\mathrm{S} 1$ ) can be exploited to obtain qualitative information on the pore size distributions in the formed gels $[22,23]$. For C100, MS20C80 and MS50C50, a broad distribution is observed ascribable to the presence of interlayer spaces, gel pores, and interhydrate pores in the $\mathrm{C}-\mathrm{S}-\mathrm{H}$ binder phase. On the other hand, for MS100 and MS80C20 well separated and relatively narrow distributions are observed. An estimate of pore dimensions was obtained for C100 and MS100 by low temperature DSC (see Supplementary material). Pores with size in the 1-3 nm range were found for both samples, while pores with dimensions between 3 and $12 \mathrm{~nm}$ were also determined for MS100.

\section{Conclusions}

Monitoring the evolution of the state of water in pastes of $\mathrm{MgO}$-based cement, Portland cement, and their mixtures by ${ }^{H} \mathrm{~N}$ NR relaxometry gave important information on the kinetics of the hydration reaction and on the formation of the porous structure of the hydrated phases. In particular, we found that the hydration of $\mathrm{MgO} / \mathrm{SiO}_{2}$ has a very short 
induction period, although consumption of water during the nucleation and growth period is slower than for Portland cement. The presence of fumed silica accelerates the hydration of Portland cement, both in the induction and nucleation and growth stages. The hydration kinetics is reflected into the evolution of the porous structure of the formed hydrated phases. For Portland cement and mixtures forming C-S-H as the main binder phase, a substantial change in pore size distributions occurs during the paste hardening due to the massive formation of $\mathrm{C}-\mathrm{S}-\mathrm{H}$ with water in the interlayer spaces and in gel pores. On the other hand, for $\mathrm{MgO} / \mathrm{SiO}_{2}$ and its mixture with Portland for which M-S-H forms as binder phase, a more gradual and less pronounced evolution of the porous structure is observed during hydration. In particular, for $\mathrm{M}-\mathrm{S}-\mathrm{H}$, the formation of the gel microstructure seems to be governed by the original spaces between $\mathrm{MgO}$ and silica particles and within silica aggregates and agglomerates, into which water can enter promoting magnesium silicate precipitation by dissolution of the reactants and transport of ions. The final pore distribution is compatible with the globular structure of M-S-H proposed by Chiang et al. [8].

\section{Acknowledgments}

This work was supported by Ministero dell'Istruzione, Università e Ricerca scientifica MIUR (FIR2013 Project RBFR132WSM).

\section{References}

[1] S.A. Walling, J.L. Provis, Chem. Rev. 116 (2016) 4170-4204, and references therein.

[2] D.R.M. Brew, F.P. Glasser, Cem. Concr. Res. 35 (2005) 85-98.

[3] Z. Li, T. Zhang, J. Hu, Y. Tang, Y. Niu, J. Wei, Q. Yu, Constr. Build. Mater. 61 (2014) 252-259. 
[4] C. Roosz, S. Grangeon, P. Blanc, V. Montouillot, B. Lotenbach, P. Henocq, E. Giffaut, P. Vieillard, S. Gaboreau, Cem. Concr. Res. 73 (2015) 228-237.

[5] S.A. Walling, H. Kinoshita, S.A. Bernal, N.C. Collier, J.L. Provis, Dalton Trans. 44 (2015) 8126-8137.

[6] B. Lotenbach, D. Nied, E. L'Hôpital, G. Achiedo, A. Dauzères, Cem. Concr. Res. 77 (2015) 60-68.

[7] M. Tonelli, F. Martini, L. Calucci, E. Fratini, M. Geppi, F. Ridi, S. Borsacchi, P. Baglioni, Dalton Trans. 45 (2016) 3294-3304.

[8] W.-S. Chiang, G. Ferraro, E. Fratini, F. Ridi, Y.-Q. Yeh, U-S. Jeng, S.-H. Chen, P. Baglioni, J. Mater. Chem. A 2 (2014) 12991-12998.

[9] P. Le, E. Fratini, K. Ito, Z. Wang, E. Mamontov, P. Baglioni, S-H. Chen, J. Colloid Interf. Sci. 469 (2016) 157-163.

[10] H.M. Jennings, Cem. Concr. Res. 38 (2008) 275-289.

[11] J.-P. Korb, Curr. Opinion Colloid Interface Sci. 14 (2009) 192-202.

[12] P.F. Faure, S. Rodts, Magn. Reson. Imaging 26 (2008) 1183-1196.

[13] V. Bortolotti, P. Fantazzini, R. Mongiorgi, S. Sauro, S. Zanna, Cem. Concr. Res. 42 (2012) 577-582.

[14] A. Valori, P.J. McDonald, K.L. Scrivener, Cem. Concr. Res. 49 (2013) 65-81.

[15] J. Greener, H. Peemoeller, C. Choi, R. Holly, E.J. Reardon, C.M. Hansson, M.M. Pintar, J. Am. Ceram. Soc. 83 (2000) 623-627.

[16] V. Bortolotti, R.J.S. Brown, P. Fantazzini, M. Mariani, Micropor. Mesopor. Mater. 178 (2013) 108-112.

[17] V. Bortolotti, L. Brizi, R.J.S. Brown, P. Fantazzini, M. Mariani, Langmuir 30 (2014) 10871-10877.

[18] P.J. McDonald, V. Rodin, A. Valori, Cem. Concr. Res. 40 (2010) 1656-1663.

[19] A.C.A. Muller, K.L. Scrivener, A.M. Gajewicz, P.J. McDonald, Micropor. Mesopor. Mater. 178 (2013) 99-103. 
[20] A. Pop, C. Badea, I. Ardelean, Appl. Magn. Reson. 44 (2013) 1223-1234.

[21] M.W. Bligh, M.N. d'Eurydice, R.R. Lloyd, C.H. Arns, T.D. Waite, Cem. Concr. Res. 83 (2016) 131-139.

[22] K.R. Brownstein, C.E. Tarr, Phys. Rev. A 19 (1979) 2446-2453.

[23] W.P. Halperin, J.-Y. Jehng, Y.-Q. Song, Magn. Reson. Imaging 12 (1994) 169173.

[24] M. Gombia, V. Bortolotti, B. De Carlo, R. Mongiorgi, S. Zanna, P. Fantazzini, J. Phys. Chem. B 114 (2010) 1767-1774.

[25] A.C.A. Muller, K.L. Scrivener, A.M. Gajewicz, P.J. McDonald, J. Phys. Chem. C 117 (2013) 403-412.

[26] S. Meiboom, D. Gill, Rev. Sci. Instrum. 29 (1958) 688-691.

[27] F. Martini, M. Tonelli, L. Calucci, M. Geppi, F. Ridi, S. Borsacchi, Cem. Concr. Res., submitted.

[28] M. Tonelli, F. Martini, L. Calucci, M. Geppi, S. Borsacchi, F. Ridi, Phys. Chem. Earth, in press DOI: 10.1016/j.pce.2017.01.011.

[29] G.C. Borgia, R.J.S. Brown, P. Fantazzini, J. Magn. Reson. 132 (1998) 65-77.

[30] G.C. Borgia, R.J.S. Brown, P. Fantazzini, J. Magn. Reson. 147 (2000) 273-285.

[31] V. Bortolotti, R.J.S. Brown, P. Fantazzini UpenWin: A software for inversion of multiexponential decay data for Windows system (C) 2012 Alma Mater Studiorum Università di Bologna, http://software.dicam.unibo.it/upenwin\%20.

[32] Mathematica, Version 10; Wolfram Research, Inc.: Champaign, IL, 2010.

[33] S. AnMing, Y. Wu, Sci. China-Tech. Sci. 53 (2010) 1471-1476.

[34] V.M. Gun'ko, I.F. Mironyuk, V.I. Zarko, E.F. Voronin, V.V. Turov, E.M. Pakhlov, E.V. Goncharuk, I.M. Nychiporuk, N.N. Vlasova, P.P. Gorbik, O.A. Mishchuk, A.A. Chuiko, T.V. Kulik, B.B. Palyanytsya, S.V. Pakhovchishin, J. Skubiszewka-Zięba, W. Janusz, A.V. Turov, R. Leboda, J. Colloid Interf. Sci. 289 (2005) 427-445. 
[35] F. Ridi, E. Fratini, P. Luciani, F. Winnefeld, P. Baglioni, J. Colloid Interf. Sci. 364 (2011) 118-124.

[36] A.C.A. Muller, K.L. Scrivener, J. Skibsted, A.M. Gajewicz, P.J. McDonald, Cem. Concr. Res. 74 (2015) 116-125.

[37] G. Land, D. Stephan, J. Mater. Sci. 47 (2012) 1011-1017.

[38] L.P. Singh, S.R. Karade, S.K. Bhattacharyya, M.M. Yousuf, S. Ahalawat, Constr. Build. Mater. 47 (2013) 1069-1077.

[39] D. Kong, Y. Su, X. Du, Y. Yang, S. Wei, S.P. Shah, Constr. Build. Mater. 43 (2013) 557-562.

[40] L.P. Singh, A. Goel, S.H. Bhattacharyya, G. Mishra, Front. Struct. Civ. Eng. 10 (2016) 162-167. 


\section{Figure Captions}

Figure 1. Representative selection of ' $\mathrm{H}^{\mathrm{T}} \mathrm{T}_{2}$ relaxation time distributions from CPMG experiments at different hydration times. Vertical expansions of these distributions are reported in Figure S1.

Figure 2. Evolution of ' $\mathrm{H} \mathrm{T}_{2}$ relaxation times from $\mathrm{CPMG}$ experiments as a function of hydration time. Symbols $\bullet$ and $\boldsymbol{\square}$ are for the more mobile and less mobile free water components, while symbol $\square$ is for free water at longer hydration times, becoming water in the interhydrate spaces. 

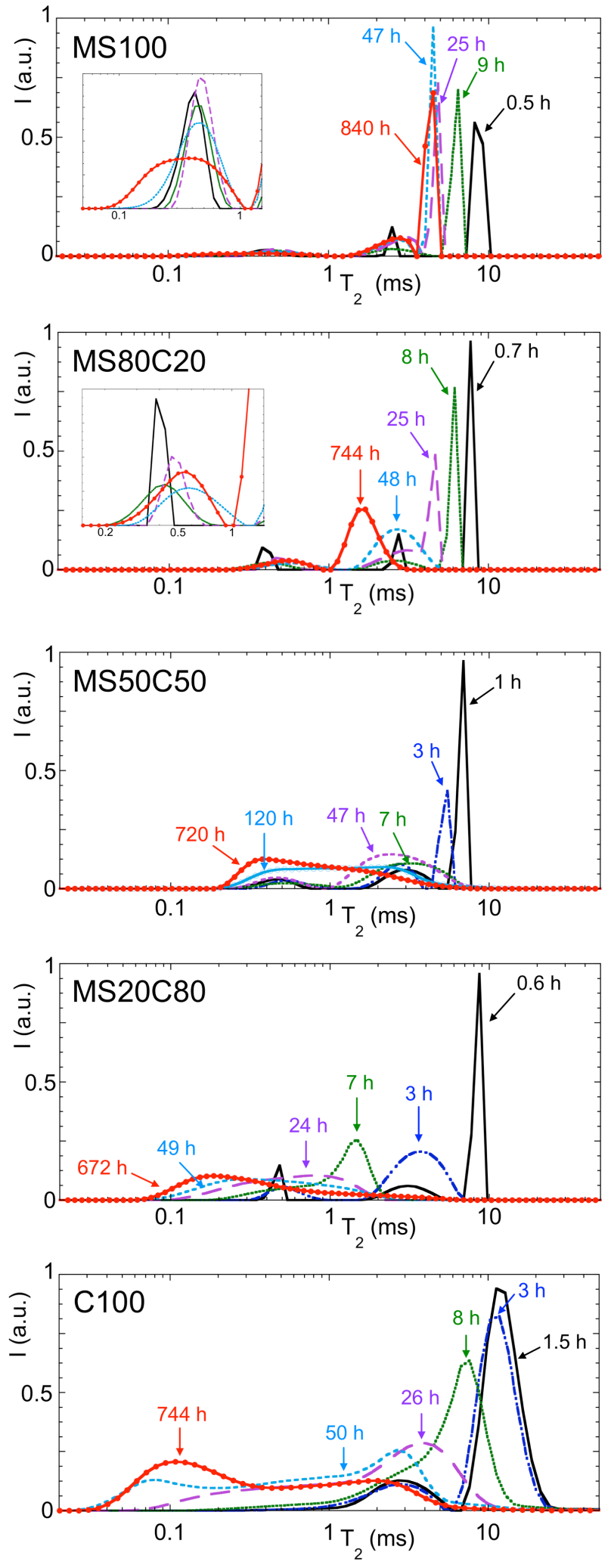

FIGURE 1 

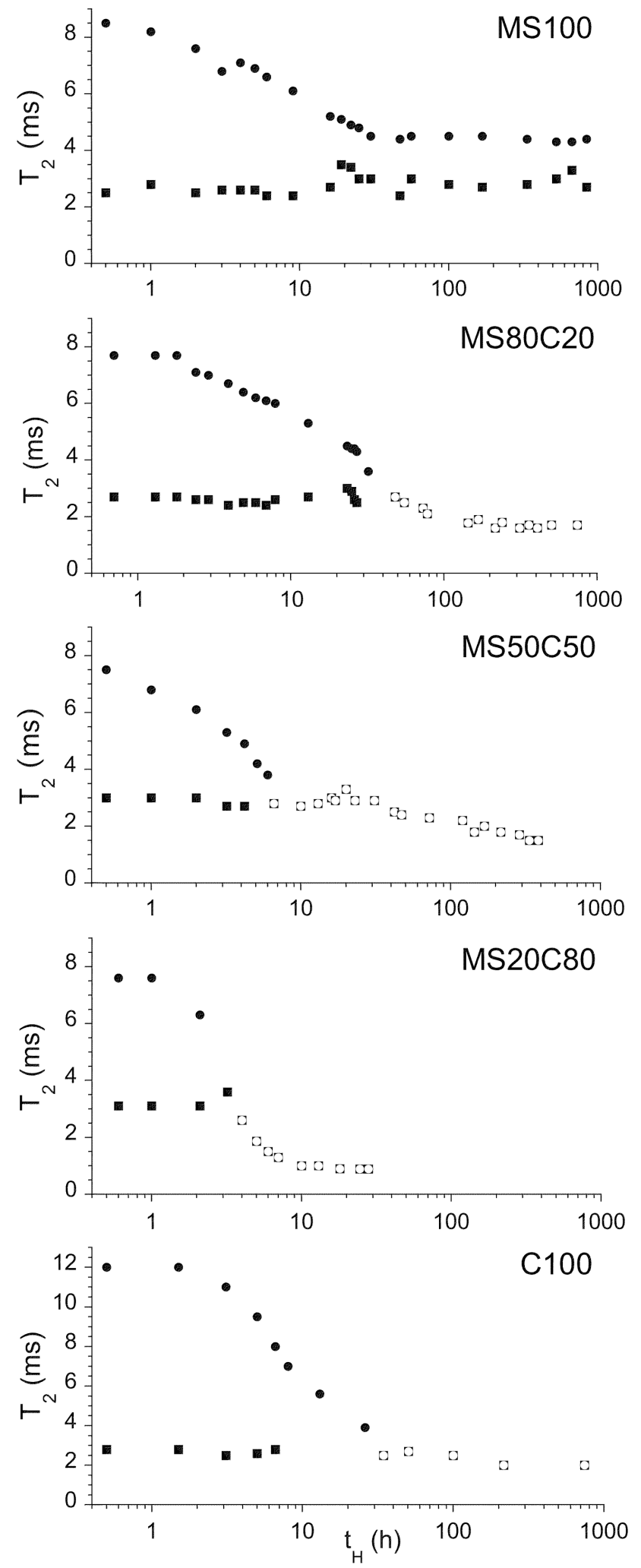

FIGURE 2 